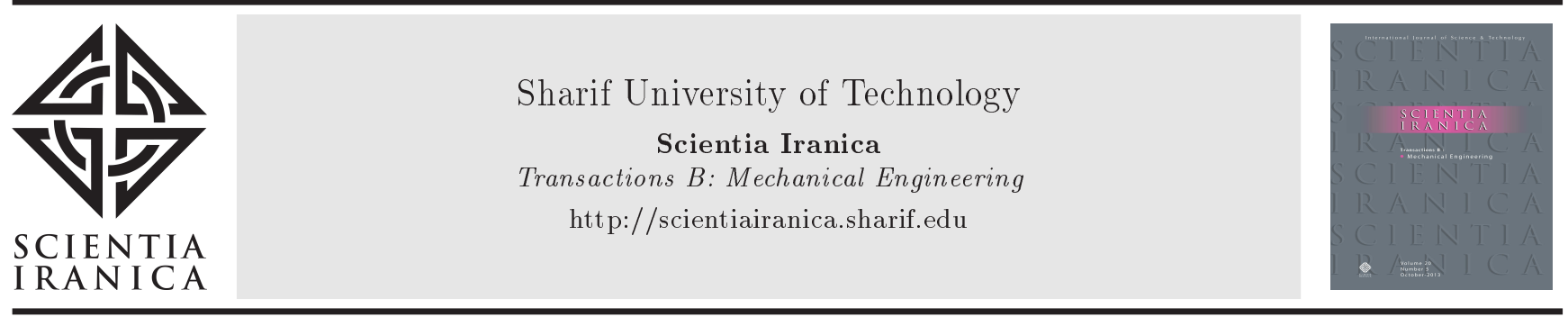

\title{
Developing a multi-objective multi-disciplinary robust design optimization framework
}

\author{
M.N.P. Meibody ${ }^{a}$, H. Naseh ${ }^{a, *}$, and F. Ommi ${ }^{b}$ \\ a. Aerospace Research Institute, Ministry of Science, Research and Technology, Tehran, Iran. \\ b. Department of Mechanical Engineering, Tarbiat Modares University, Tehran, P.O. Box 14665-834, Iran. \\ Received 25 January 2020; received in revised form 13 July 2020; accepted 10 March 2021
}

\author{
KEYWORDS \\ Multi-disciplinary \\ Design Optimization \\ (MDO); \\ Robust Design \\ Optimization (RDO); \\ Bi-Level Integrated \\ System Synthesis \\ (BLISS); \\ Progressive Latin \\ Hypercube Sampling \\ (PLHS); \\ Hydrazine \\ monopropellant \\ thruster.
}

\begin{abstract}
This study aims to provide an efficient Multi-Objective Multidisciplinary Robust Design Optimization (MOMRDO) framework. To this end, Bi-Level Integrated System Synthesis (BLISS) framework was implemented as a fast Multi-disciplinary Design Optimization (MDO) framework. Progressive Latin Hypercube Sampling (PLHS) was developed as a Design Of Experiment (DOE) for the Uncertainty Analysis (UA). This systematic approach leads to a fast, adaptive, and efficient Robust Design Optimization (RDO) framework of complex systems. The accuracy and performance of the proposed algorithm have been evaluated through various tests. Finally, the RDO of a hydrazine monopropellant thruster was selected as a case study. The results showed that this method is a fast and effective method for the multi-objective optimization design of complex systems, and it can also be used in other engineering applications.
\end{abstract}

(C) 2021 Sharif University of Technology. All rights reserved.

\section{Introduction}

Nowadays, as the system matures, the use of Multidisciplinary Design Optimization (MDO) in the design process has become commonplace [1]. Although the concept of MDO was first introduced in aerospace engineering [2,3], its application in the design of other complex systems (such as electrical machines, automobiles [4], robots [5], and tunnel boring machines) has rapidly expanded. On the other hand, exposure to uncertainties in the life cycle of a product is inevitable. These uncertainties affect product performance and

*. Corresponding author. Tel: $(+98) 88366030$

E-mail address: hnaseh@ari.ac.ir (H. Naseh)

doi: $10.24200 /$ sci.2021.55306.4159 functional constraints. In complex systems, the impact of uncertainty is more important. This feature became a source of inspiration for the concept of Uncertain Multidisciplinary Robust Design Optimization (UMRDO) in the optimal design of complex systems. Generally, two approaches are applied to solve such problems including the Reliability-Based Design Optimization (RBDO) and Robust Design Optimization (RDO). RBDO reduces the failure risks, while RDO reduces the sensitivity of the responses $[6,7]$.

Many efforts have been made to provide Multidisciplinary Robust Design Optimization (MRDO) by integrating Uncertainly Analysis (UA) into the MDO frameworks $[1,8-10]$. In all cases, the MRDO has been built by combining the common UA approaches and the MDO framework. However, the computational cost of MRDO is still the main challenge for such 
problems. Thus, the MRDO study moves into a new age of research.

Generally, the UA methods are divided into two kinds of deterministic analysis and non-deterministic analysis. In the case of problems with a large number of uncertain parameters and/or nonlinear responses, the utilization of non-deterministic methods is inevitable [11]. The probability theory is one of the conventional non-deterministic UA approaches. The principle of using UA probability theory is based on random sampling.

Monte Carlo Simulation (MCS) is the first and most common sampling method for UA. Thus, in some cases, UA is also referred to as MCS. The bigger simulation of the sample size leads to more accuracy in the MCS outputs. Studies have shown that the standard MCS despite its simplicity is inefficient [12]. It is because of its inability to fill the space and its collapse properties.

In the past few decades, many efforts have been made to improve sampling efficiency. Variance Reduction Technique (VRT) is considered to be the most effective approach to improve sampling efficiency $[13,14]$. Although VRT increases sampling efficiency, it does not use the minimum sample size of UA [15]. Progressive Sampling Techniques (PST) solves the problem of finding the least possible value of samples. Fundamentally, PST works by training a learning algorithm, and then the sample size will continue to grow until the desired accuracy is achieved. Simplicity in application and efficiency in sample generation is the most important feature of PST. Through combining the VRT and PST an optimal sample size could be obtained.

Latin Hypercube Sampling (LHS), as a stratified sampling method to ensure that the sample will not collapse. Similarly, the improved LHS method can fill the space to the greatest extent. Some activities were performed to convert LHS to Progressive Latin Hypercube Sampling (PLHS). The most cited PLHS strategies are the Doubling Procedure (DP) and Sliced Latin Hypercube Sampling (SLHS) [16]. The first method, DP, doubles the sample size in each step. Despite the simplicity of its performance, DP diminishes the flexibility of the sample size [17]. This lowers the efficiency of sampling. SHLS was originally developed by Qian [18] and further enhanced by other scientists [19]. SLHS generates a series of smaller subsets. The SLHS result is quasi-LHS.

The main contribution of this paper is to propose a novel MRDO framework based on fast and effective MDO and UA methodology. To this end, Bi-Level Integrated System Synthesis (BLISS) was implemented as a fast MDO framework. Similarly, UA was performed by the PLHS technique of variable sample size.

\section{Multi-Objective Robust Multidisciplinary Design Optimization (MOMRDO) framework}

Implementing a novel multi-disciplinary RDO framework, which is a combination of MDO and RDO, was evaluated. Generally, the RDO problem was defined as Eq. (1):

$$
\begin{aligned}
& \text { find: } x \\
& \text { minimize: } \phi\left[f\left(x, \sigma_{x}\right), \sigma\left(x, \sigma_{x}\right)\right] \\
& \text { subjected to: } g_{i}(x) \leq 0
\end{aligned}
$$

where $x$ represents the design variables; and $\phi$ is the robust optimization objective that is a function of the problem objective function $f\left(x, \sigma_{x}\right)$ and the objective function deviation $\sigma\left(x, \sigma_{x}\right)$.

As shown in Eq. (1), the design variables were extracted so that the robust optimization objective function was minimized in the face of uncertainties. Such a process requires coordinated optimization and simulation and analysis of uncertainties. As shown in Figure 1, the proposed process is an iterative method resulting from the combination of multi-disciplinary optimization and uncertainty analysis.

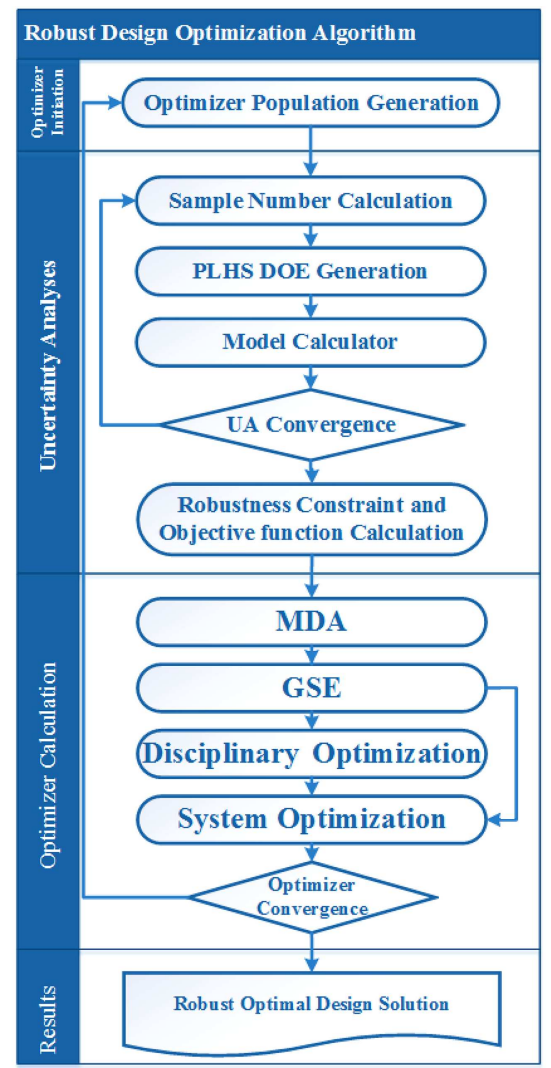

Figure 1. Multi-Objective Robust Multidisciplinary Design Optimization (MORMDO) flowchart. 


\subsection{BLISS optimization framework}

The BLISS framework was proposed by Sobieski in 1998 as the MDO framework [20]. As the name suggests, in this framework, optimization problems are divided into two levels: system and subsystem. A schematic flowchart of the multi-disciplinary optimization of this framework is presented in the optimizer section of Figure 1. This framework uses MultiDisciplinary Analysis (MDA) to find the coupling variables and uses the General Sensitivity Equation (GSE) to find the derivatives of the functions to calculate the objective functions at the subsystem level and the system level and updates design variables at each iteration [21].

\subsection{Uncertainty analyses algorithm}

In the RDO issue, the main challenge is to define and calculate the uncertainty propagation. The UA method based on statistical simulation provides an effective solution for a wide range of applications [22-25]. In this study, the PLHS-based UA was carried out.

\subsubsection{UA algorithm}

The UA algorithm based on PLHS includes five steps while are repeated to satisfy convergence condition. The proposed UA algorithm is described in Table 1.

In Eq. (2), $E$ and $n_{i}$ are the output variation and iteration sample size, respectively; $K$ is the preexponential factor; and $\tau$ is the population correction factor. In each iteration, these factors were computed and updated based on the responses of the previous three iterations.

$$
E_{(\text {mean }, \sigma)_{i-1}}=K e^{-\tau n_{i}}
$$

\subsubsection{PLHS mathematical definitions}

PLHS with normal and uniform distribution was used for the design parameters to extract the population needed for the UA and Sensitivity Analyses (SA), respectively. To generate $n$ samples of $m$ parameters with uniform distribution (or any desired distribution) within the parameters definition interval, a hypercube of unit $m$ dimensions, $H^{m}$, was first defined by Eq. (3):

$$
H^{m}=[0,1]^{m} .
$$

Each dimension of the unit hypercube was divided equally (or proportional to the distributive distributions) into $n$ part of Eq. (4):

$$
H_{j}=\left\{\left[0, \frac{1}{n}\right],\left[\frac{1}{n}, \frac{2}{n}\right], \ldots,\left[\frac{n-1}{n}, 1\right]\right\}=\bigcup_{i=1}^{n} H_{j}^{i}
$$

According to the definition given in Eq. (5), the samples are randomly designed under condition of projection property:

$$
x_{i}=\left\{x_{i, 1}, x_{i, 2}, \ldots, x_{i, m}\right\} .
$$

To check the samples projection property a set of auxiliary binary variables was defined as $y_{i, j}$ such that:

$$
y_{i, j}= \begin{cases}1 & x_{i, j} \in H_{j}^{i} \\ 0 & x_{i, j} \notin H_{j}^{i}\end{cases}
$$

The $n \times m$ sample matrix, $S(m, n)$, was defined with the $x_{i j}$ entries as Eq. (7):

$$
S(m, n)=\left[\begin{array}{ccc}
x_{11} & \ldots & x_{1 m} \\
\cdot & x_{i j} & \cdot \\
x_{n 1} & \ldots & x_{n m}
\end{array}\right]
$$

Then $S(m, n)$ was said to be Latin Hypercube if and only if the following condition was satisfied.

$$
\frac{\sum_{j=1}^{m} \sum_{i=1}^{n} y_{i, j}}{n * m}=1
$$

The left-hand side of Eq. (8) varied in the interval $\left[\frac{1}{m}, 1\right]$. To increase the sample number to $n^{\prime}>n$, first, each dimension of the unit hypercube should be divided into $n^{\prime}$ sections. The samples inherited from the previous step are then placed in the space of the new divisions. The new samples were produced in such a way that the maximum value could be obtained using the left-hand side of Eq. (8). The new sample matrix was formed by the combination of the new sample and the inherited sample. In each step, the new sample matrix was LHS. An example of the PLHS process is shown in Figure 2.

Table 1. Process of PLHS-based Uncertainty Analysis (UA).

- DOE generation of PLHS-based design variables for UA;

- Objective functions responses extraction based on generated DOE;

- Calculating the mean and standard deviation of the responses;

- Calculating the normalized variations of the mean and standard deviation from the previous iteration values;

- Convergence test: For the case where the deviation is greater than 2\%, a new set of DOE was generated by increasing the sample size in proportion to the variable, as shown in Eq. (2) 

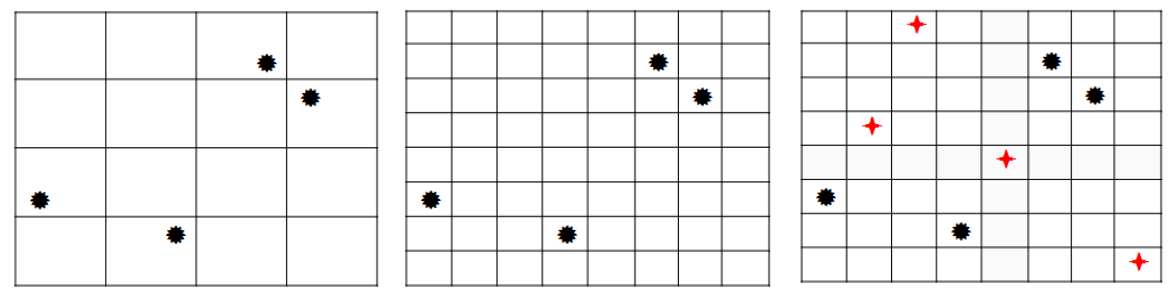

Figure 2. An example of Progressive Latin Hyper Cube Sampling (PLHS) process.

\section{Results and discussions}

The validations of Design Of Experiment (DOE) and MDRDO algorithms are presented in this section. Also, the result of the design of optimization results of the case study is discussed.

\subsection{Verification}

To evaluate the performance of the proposed MRDO algorithm, some experiments were carried out. The computational cost advantage of the proposed method over Monte Carlo and other sampling-based simulations has also been evaluated.

\subsubsection{Validation of BLISS algorithm}

To validate the performance of the developed optimizer, an analytic multidisciplinary problem needs to be optimized. Therefore, Eq. (9) was implemented on the BLISS framework. Yi et al. have solved this problem [26]. It included two disciplines. Each discipline had a state variable. Each of these state variables was also a dependent variable. In the first discipline, there were two global design variables and one additional local design variable. The relationship between disciplines was nonlinear. Each discipline had a local constraint.

$$
\text { minimize } x_{1}^{2}+x_{2}+y_{1}+e^{-y_{2}}
$$

where:

$$
\begin{aligned}
& y_{1}=z_{1}^{2}+x_{1}+x_{2}+0.2 y_{2}, \\
& y_{2}=z_{1}+x_{2}+\sqrt{y_{1}} \\
& g_{1}=1-\frac{y_{1}}{3.16} \leq 0 \\
& g_{2}=\frac{y_{2}}{24}-1 \leq 0 \\
& -10 \leq z_{1} \leq 10 \\
& 0 \leq x_{1} \leq 10 \\
& 0 \leq x_{2} \leq 10 .
\end{aligned}
$$

These results were in agreement with the findings reported by Perez et al. [27].

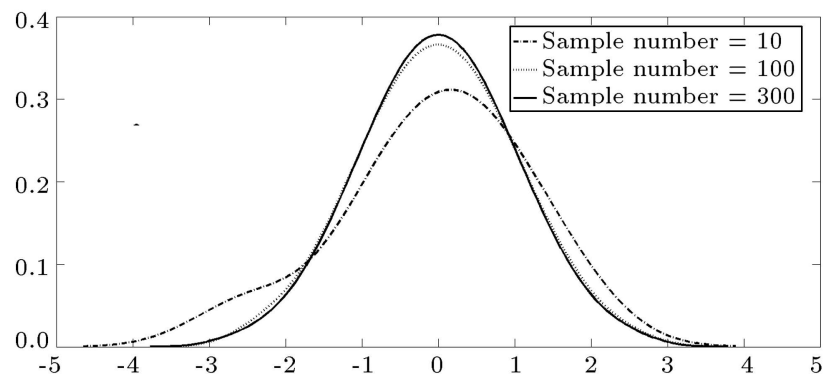

Figure 3. Sample number effects on the sampling distribution.

\subsubsection{Sampling distribution justification}

LHS is a sampling method whose output results have a uniform distribution feature. To achieve samples with non-uniform distribution, it is necessary to use an appropriate mapping through the division of the unit hypercube. Therefore, it is necessary to verify the performance of the required mapping. To study the distribution of PLHS results, the Probability Density Function (PDF) of three DOEs were evaluated. Each DOE was designed to produce samples with a normal distribution with a mean of 0 and a standard deviation of 1. The DOE sample size increased in two steps, from 10 to 100 and from 100 to 300. The PDFs of these DOEs are shown in Figure 3.

The results shown in Figure 3 demonstrate the normal distribution of the sample. In addition, the sample distribution was similar to the normal distribution and the simulation accuracy increased by increasing the sample size. As shown in Figure 3, by increasing the sample size, the PDE diagram became more similar to the normal distribution.

\subsubsection{Sampling convergence characteristics}

In the case of different sample sizes, by comparing the MCS and PLHS, a mean value of 1 and a standard deviation of 0.5 were obtained. Figures 4 and 5 show the changes in the sample size of the mean and standard deviation, respectively. The results showed that the convergence rate of PLHS was faster than that of MCS.

\subsubsection{Algorithm verification}

The proposed RDO performance was verified by the implementation of the Sinc function as the benchmark. The definition of the Sinc function is given in Eq. (10). 


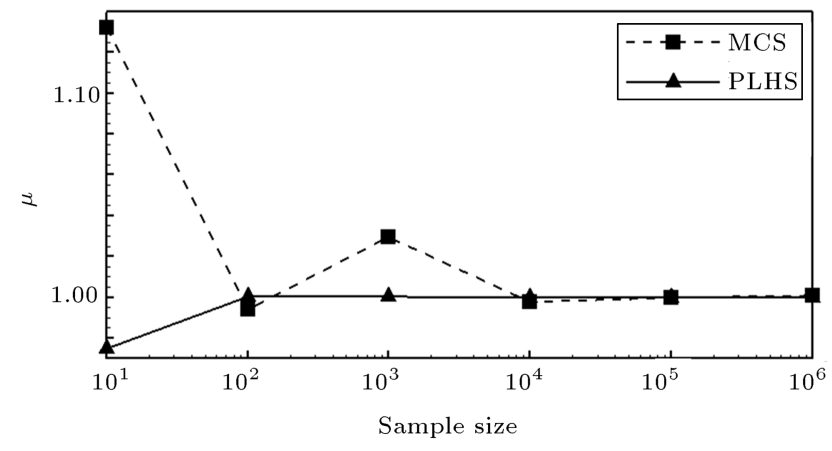

Figure 4. The relationship between the mean value and sample size for Monte Carlo Simulation (MCS) and Progressive Latin Hyper Cube Sampling (PLHS) methods.

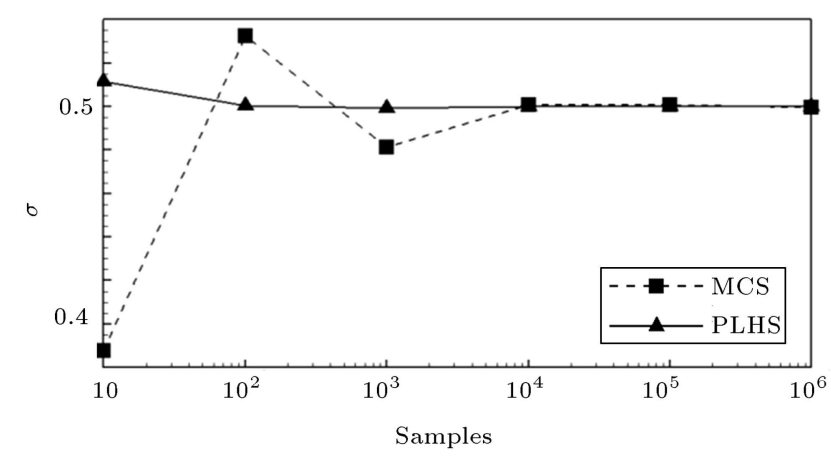

Figure 5. The relationship between the standard deviation and sample size for Monte Carlo Simulation (MCS) and Progressive Latin Hyper Cube Sampling (PLHS) methods.

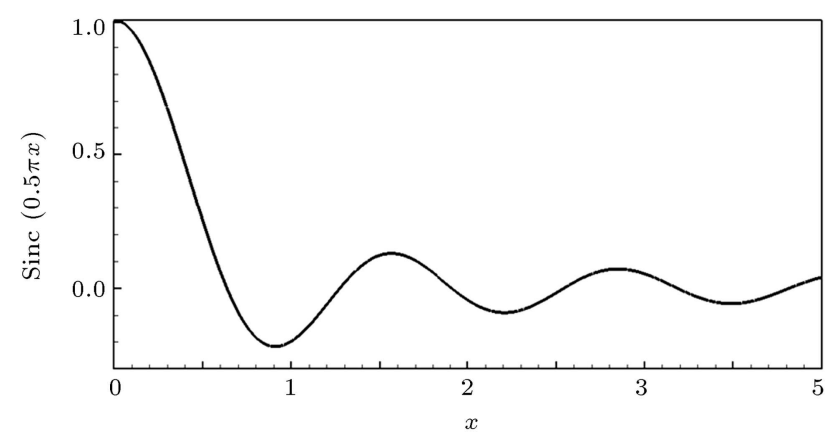

Figure 6. Sinc function.

The Sinc $(0.5 \pi x)$ function is plotted in Figure 6 .

$$
\operatorname{Sinc}(x)=\frac{\sin (x)}{x}
$$

Sinc function included multiple local optimums. Furthermore, the robustness values of these points were different. Verification of the RDO algorithm was carried out by varying the standard deviation of the design parameter from zero to a half. The objective function of the RDO was calculated as the sum of mean and standard deviation values of the Sinc function. The Sinc function robust optimal points versus the standard

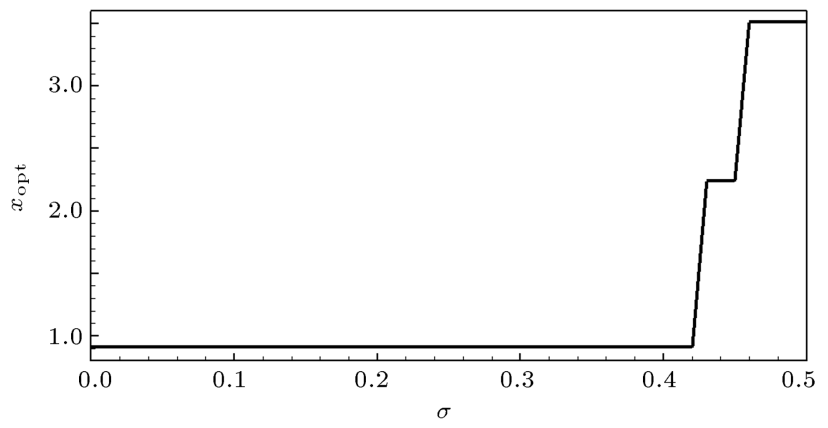

Figure 7. The Sinc function robust optimal points versus the standard design parameter deviation.

design parameter deviation are shown in Figure 7. Due to the nature of the Sinc function, the robustness of the RDO objective function varies by choosing different local optimum points.

\subsubsection{Computational cost}

The computational time of various UA approaches to implement RDO was compared. These used UA approaches are MCS, Sobol, LHS, SLHS, and PLHS. The standard deviation of the design parameter was considered to be 0.5 units. To ensure the accuracy of RDO, each method was run 100 times, and then the average run time was used to extract the solving time. Table 2 lists the computational cost of all simulationbased RDOs. The computational time of the PLHSbased RDO was close to one-third of MCS one. The detailed information of the simulations is provided in the Supplementary Data.

To evaluate the accuracy of the proposed RDO with small sample size, the MCS and PLHS-based RDO was compared. The results of this comparison are presented in Table 3. As shown in the table, the error rate of MCS was $27 \%$. The results of the study of computational cost indicated that although the use of advanced sampling methods reduces the problemsolving time by reducing the number of samples, in RDO with the same number of samples for different sampling methods, simpler sampling approaches lead to a lesser solution time. It is worth noting that in the simpler sampling method, the smaller the number of samples, the lower the accuracy of RDO.

Table 2. Robust Design Optimization (RDO) computational cost based on sampling.

\begin{tabular}{cccc}
\hline Method & Optimal point & Samples & Time $(\mathrm{s})$ \\
\hline MCS & 3.5 & $1 \mathrm{e}+4$ & 14.18 \\
Sobol & 3.5 & $5 \mathrm{e}+3$ & 7.32 \\
LHS & 3.5 & $1 \mathrm{e}+3$ & 6.2 \\
SLHS & 3.5 & $(250-430)$ & 4.17 \\
PLHS & 3.5 & $(190-375)$ & 3.78 \\
\hline
\end{tabular}


Table 3. Comparison of robust optimization results of Monte Carlo Simulation (MCS) and Progressive Latin Hyper Cube Sampling (PLHS) based on 500 samples.

\begin{tabular}{cccc}
\hline Method & $\begin{array}{c}\text { Optimal } \\
\text { point }\end{array}$ & $\begin{array}{c}\text { Time } \\
(\mathbf{s})\end{array}$ & $\begin{array}{c}\text { Correctness } \\
(\%)\end{array}$ \\
\hline MCS & 3.5 & 4.06 & 73 \\
Sobol & 3.5 & 4.08 & 86 \\
LHS & 3.5 & 4.10 & 94 \\
SLHS & 3.5 & 4.18 & 98 \\
PLHS & 3.5 & 4.23 & 100 \\
\hline
\end{tabular}

\subsection{Case study: Monopropellant thruster design optimization}

In this study, RDO of the $22 \mathrm{~N}$ monopropellant thruster was used as a case study of the proposed algorithm. The Design Structure Matrix (DSM) of a monopropellant thruster is pictured in Figure 8. Two deterministic and non-deterministic optimization methods have been implemented in the design of this thruster system. In both methods, the BLISS framework was implemented as an optimizer. In the following, the elements of deterministic and non-deterministic optimization are defined.

\subsubsection{Structure}

The task of the thruster structure is to integrate and bear the loads applied to it. On the other hand, one of the most important results of the conceptual design of each system is the estimation of its mass. In this system, the total mass was obtained by adding the masses of the Injector Head ( $\mathrm{IH})$, reaction chamber, and nozzle according to Eq. (11):

$$
M_{\text {Total }}=M_{i n j}+M_{C B}+M_{N} .
$$

The mass of subsystems was calculated based on volumetric relationships. The thickness of the structure of each segment, $t_{s u b_{i}}$, was calculated from Eq. (12). Then their volume, $V_{\left(s u b_{i}\right)}$, was extracted and placed in Eq. (13):

$$
\begin{aligned}
& t_{s u b_{i}}=\frac{n_{S F}}{\sigma}\left(\frac{\pi P_{s u b_{i}} R_{s u b_{i}}+T_{C C}}{\pi R_{s u b_{i}}^{2}}\right), \\
& M_{s u b_{i}}=\rho_{s u b_{i}} V_{s u b_{i}}=\rho_{s u b_{i}} A_{s u b_{i}} t_{s u b_{i}} .
\end{aligned}
$$

\subsubsection{Injector Head (IH)}

In the monopropellant thrusters, the IH was responsible for the uniform distribution of the propellant on the Catalyst Bed (CB). To make the propellant uniformly distributed on the catalytic surface, the swirl-axial injector with full cone injection was selected as the injector [28]. A computer program was developed to design the IH. The mass flow rate of the propellant and the diameter of the $\mathrm{CB}$ were introduced as the system input of the ejector head design. In addition, the spray angle, the number of the tangential grooves, and the inclinational angle of the grooves were introduced as local parameters of the ejector design. The pressure drop, the injector discharge coefficient, the injector orifice diameter, and the injector mass were implemented as the $\mathrm{IH}$ design responses.

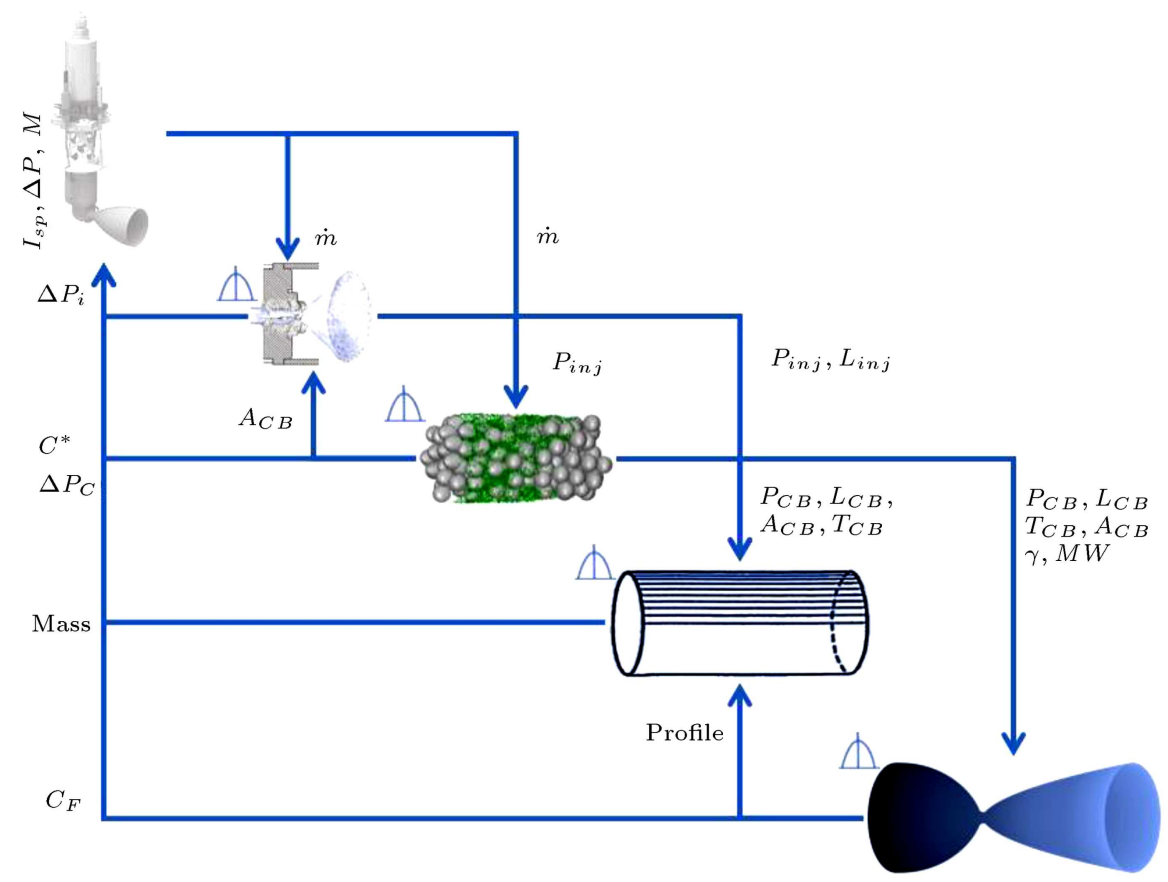

Figure 8. The monopropellant thruster design structure matrix. 


\subsubsection{Catalyst Bed ( $C B)$}

In monopropellant thrusters, the $\mathrm{CB}$ controls the decomposition of the propellant $[29,30]$. Since 1963 the most successful hydrazine decomposition catalyst had been Shell 405. In this section, the modelling and SA of the $\mathrm{CB}$ design will be introduced. With reference to [31-33], an analysis of the $\mathrm{CB}$ behavior has been derived. The Runge-Kutta Fehlberg method with a variable time step was used to calculate the $\mathrm{CB}$ model.

\subsubsection{Nozzle}

The nozzle design was performed in the closed-loop calculation to achieve a specific thrust level. The nozzle design flowchart is shown in Figure 9. This closedloop includes the geometric estimation and numerical simulation of nozzle performance. The process was arranged according to the following steps:

- The nozzle thrust coefficient was estimated through gas dynamic calculations $[34,35]$;

- According to the obtained nozzle coefficient, the nozzle throat, and outlet area were calculated [36];

- The nozzle shape was determined by a geometric method;

- The nozzle performance was evaluated through numerical simulation;

- The simulated thrust coefficient was compared with its previous calculation.

The convergence criteria should be less than $2 \%$ of the difference between the previous value and the present value of the thrust coefficient in the loop.

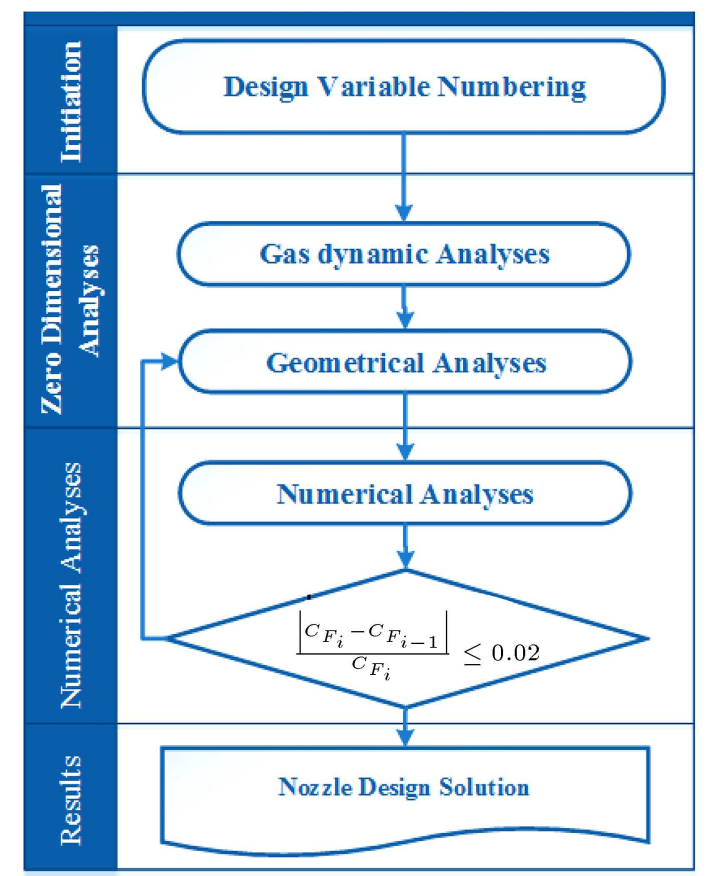

Figure 9. The nozzle design flowchart.
Gas Dynamic Analysis (GDA) ignores friction and turbulence losses and thrust vector misalignment. Hence, numerical simulation is a powerful strategy for evaluating nozzle performance. In this research, the nozzle design accuracy improved by developing the nozzle internal flow simulation during the design process. The nozzle profile was designed based on a geometrical strategy and the GDA results. This method is shown in Figure 10.

Four boundary conditions, including inlet, axial symmetry, wall, and output, were considered for the nozzle simulation. The boundary conditions are shown in Figure 11. The outlet pressure and temperature of the reaction chamber were defined under the inlet boundary condition. On the axially symmetric boundary, the vertical gradient of flow was assumed to be zero. The non-slip condition was also considered in the wall. The static pressure was presented as the output boundary condition, and the other parameters were extrapolated from inside the solution.

A fully structured mesh has been used in the nozzle numerical simulation. In the vicinity of the walls, the cells are partially scattered to capture the viscous sub-layer $\left(y^{+} \cong O(1)\right)$. An example of a convergent-divergent nozzle meshing is illustrated in Figure 12.

To perform compressible flow simulations, density-based implicit solver software (Ansys Fluent software) was used. The $k-\omega$ SST model was used as a turbulence model. In addition, continuity conservation equation, energy conservation equation, and turbulence were couple with the second-order method.

\subsubsection{Optimization problem}

The deterministic and non-deterministic optimization problems of the monopropellant thruster were formulated in the form of Eqs. (14) and (15), respectively:

$$
\begin{aligned}
& \text { Find } x=\left[\alpha_{\iota \nu \phi}, P_{C C}, \nu, n, T_{0}, D_{P}, D_{C C}, \eta_{c c}, \beta_{3}, \beta_{4}\right] . \\
& \text { Minimize } \Delta P, M_{\text {Total }} \\
& \text { Maximize } I_{s p} \\
& \text { Subject to Constraints, } \\
& \text { Find } x=\left[\alpha_{\iota \nu \phi}, P_{C C}, \nu, n, T_{0}, D_{P}, D_{C C}, \eta_{c c}, \beta_{3}, \beta_{4}\right] \\
& \text { Minimize } F_{N D e t}\left(\Delta P, M_{\text {Total }}, I_{s p}\right) \\
& \text { Subject to Constraints. }
\end{aligned}
$$

In the non-deterministic optimization problem, the objective functions and related constraints were generated by the sum of mean and standard deviations obtained from the UA. 


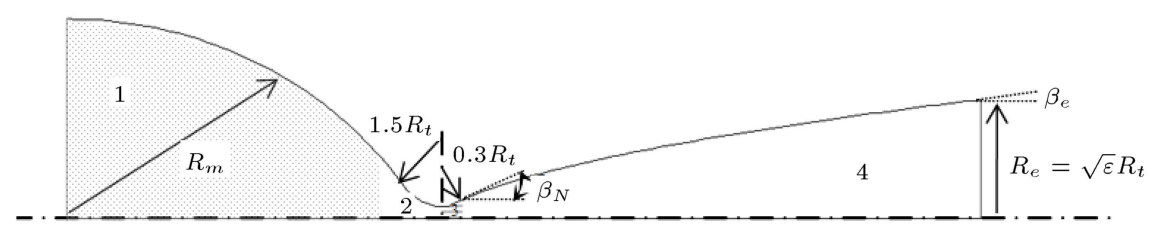

Figure 10. Schematic of nozzle profile.

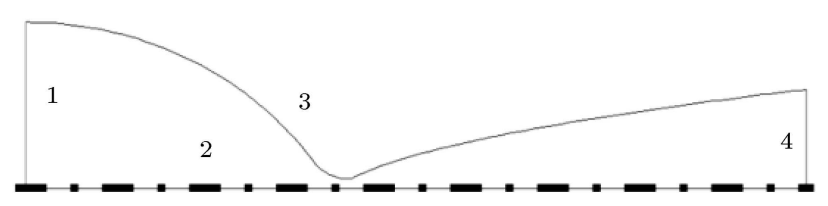

Figure 11. Schematic of nozzle boundary conditions.

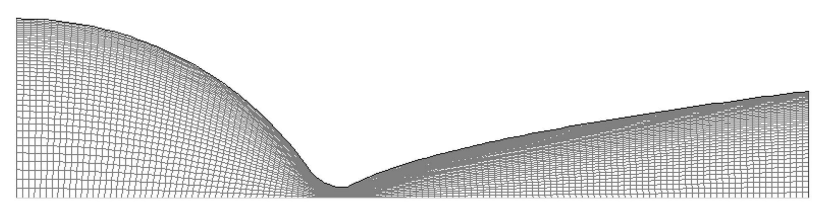

Figure 12. An example of a meshed convergent-divergent nozzle.

\subsubsection{Objective functions}

In this research, the minimization of thruster weight and pressure drop and maximization of the thruster specific impulse was considered as objective functions. The objective functions of the deterministic optimization problem were defined in the multi-objective function in the form of Eq. (16):

$$
\begin{aligned}
& F_{\text {Det }}\left(\Delta P, M_{\text {Total }}, I_{s p}\right)=-\frac{w_{1}}{200} I_{s p}+\frac{w_{2}}{300} M_{\text {Total }} \\
& \quad+\frac{w_{3}}{200} \Delta P \\
& \text { Subjected to } w_{1}+w_{2}+w_{3}=1
\end{aligned}
$$

where $w_{i}$ are variable coefficients that were defined by the total sum constraint equal to 1 in the optimization.

In non-deterministic optimization, the optimization objective function was defined as a multi-objective function in the form of Eq. (17):

$$
\begin{aligned}
F_{D e t}= & -\frac{w_{1}}{200}\left(I_{s p}+\sigma_{I_{s p}}\right)+\frac{w_{2}}{300}\left(M_{\text {Total }}+\sigma_{M_{\text {Total }}}\right) \\
& +\frac{w_{3}}{200}\left(\Delta P+\sigma_{\Delta P}\right)
\end{aligned}
$$

Subjected to $w_{1}+w_{2}+w_{3}=1$.

\subsubsection{Design parameters}

The correct selection of these variables is one of the challenges of the designers, and it is also a decisive factor for achieving the optimal realistic design with the smallest computational cost. When selecting the design variables of the optimization problem, the constraints and objective function should be affected as much as possible while describing the design problem completely. On the other hand, the design variables should be as independent as possible. For this reason, SA have been carried out on each subsystem. Figure 13 shows the Pairwise Scatter Plot Matrix (PSPM) and histogram of the design variables and the responses of the CB. The pairwise spread of $\mathrm{CB}$ design parameters means that the inputs are distributed independently of each other. In this graph, the dependence of the response from the design parameters is also specified. Among the design inputs, the reaction chamber pressure had the least effect on the bed design responses. Moreover, the SA of the CB clearly illustrated the design objectives of the $\mathrm{CB}$.

Also, in the non-deterministic optimization problems, besides the selection of the design variables selecting design variables, the identification and assigning the amount of uncertainty of each variable will affect the calculation cost and the correct optimal design achievement. As shown in Table 4, the design parameters and their uncertainties were defined according to the subsystems of the thruster.

\subsubsection{Constraints}

The maximum propellant inlet pressure, CB loading factor, $\mathrm{CB}$ aspect ratio, and the decomposition products Mach number at the nozzle inlet were introduced as design constraints in the form of Eqs. (18) to (21):

$$
\begin{aligned}
& P_{\text {in }} \leq 22 \text { bar } \\
& G_{C a t} \leq 400 \frac{\mathrm{kg}}{\mathrm{m}^{2}}, \\
& \frac{L_{C C}}{D_{C C}} \leq 2, \\
& M_{N_{i n}} \leq 0.3 .
\end{aligned}
$$

In non-deterministic optimization, the sum of the mean and standard deviation of the optimization constraints was defined as Eqs. (22) to (25):

$$
\begin{aligned}
& P_{i n}+\sigma_{P_{i n}} \leq 22 \text { bar, } \\
& G_{C a t}+\sigma_{G_{C a t}} \leq 400 \frac{\mathrm{kg}}{\mathrm{m}^{2}}
\end{aligned}
$$




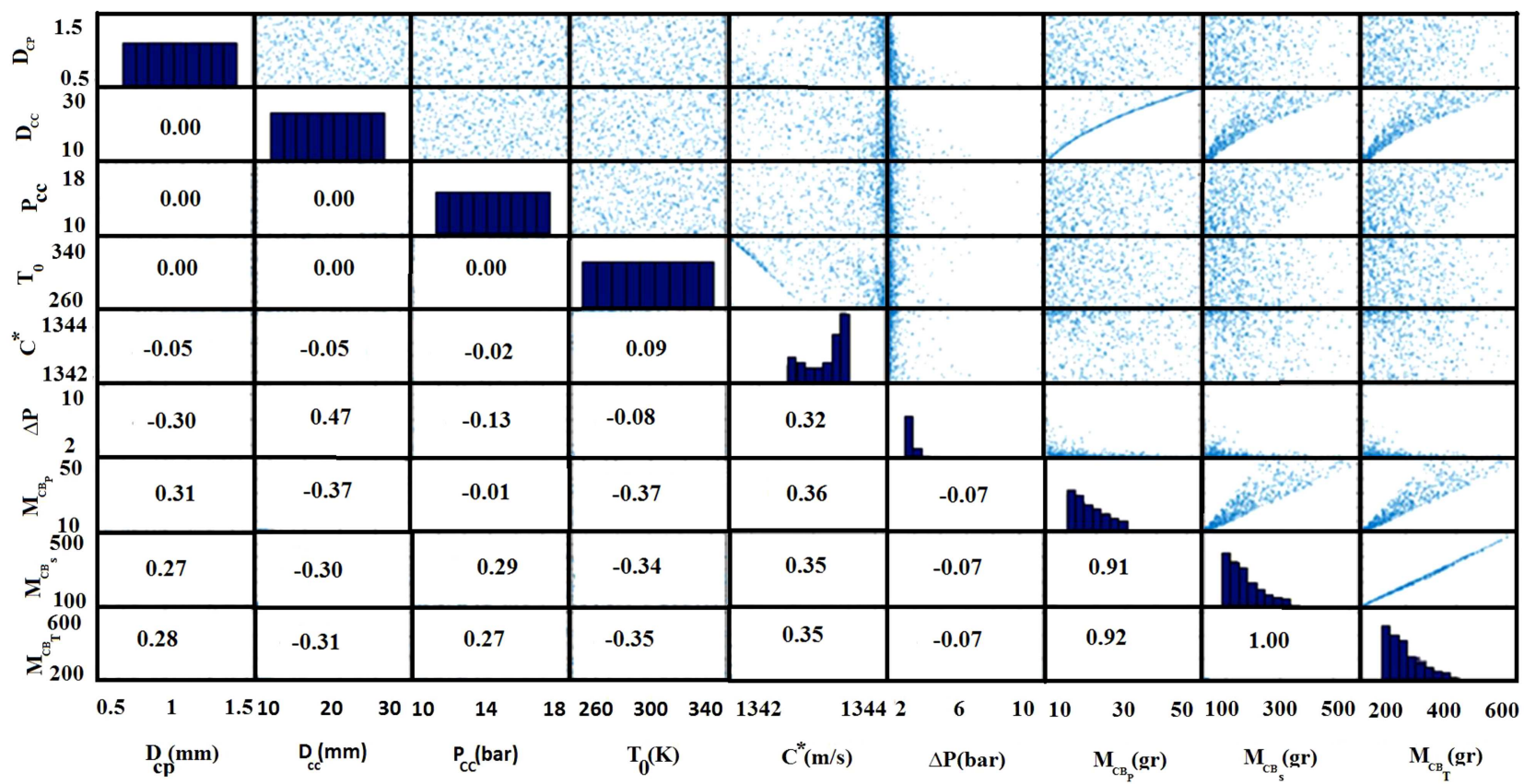

Figure 13. Pairwise Scatter Plot Matrix (PSPM) and histogram of Sensitivity Analysis (SA) for Catalyst Bed (CB).

Table 4. Design parameters, range of variations and their uncertainty definition.

\begin{tabular}{|c|c|c|c|c|c|c|c|c|}
\hline \multicolumn{3}{|c|}{ Variable } & \multicolumn{2}{|c|}{ Range } & \multirow[t]{2}{*}{ Unit } & \multirow[t]{2}{*}{ Variable type } & \multirow[t]{2}{*}{$\sigma$} & \multirow[t]{2}{*}{ Uncertainty type } \\
\hline & & & $\min$ & $\max$ & & & & \\
\hline \multirow{2}{*}{ Global } & Reaction chamber pressure & $P_{C C}$ & 5 & 20 & bar & Continues & $\% 10$ & Normal \\
\hline & Catalyst bed diameter & $D_{C C}$ & 10 & 40 & $\mathrm{~mm}$ & Continues & $\% 0.2$ & Normal \\
\hline \multirow{11}{*}{ Local } & Spray angle & $\alpha$ & 60 & 90 & $\circ$ & Continues & $3^{\circ}$ & Normal \\
\hline & Grooves inclinational angle & $\nu$ & 10 & 35 & $\circ$ & Continues & $1^{\circ}$ & Normal \\
\hline & Number of grooves & $n$ & 3 & 5 & \# & Discrete & - & - \\
\hline & Inlet propellant temperature & $T_{0}$ & 250 & 350 & $\mathrm{~K}$ & Continues & $10 \mathrm{~K}$ & Normal \\
\hline & Catalyst particle diameter & $D_{P}$ & 0.5 & 2 & $\mathrm{~mm}$ & Continues & $\% 10$ & Normal \\
\hline & Thermo-chemical efficiency & $\eta_{C C}$ & 0.85 & 0.97 & - & Continues & $\% 2$ & Normal \\
\hline & Nozzle expansion ratio & $\varepsilon_{N}$ & 2 & 100 & - & Continues & - & - \\
\hline & Nozzle's converges angle & $\beta_{3}$ & 20 & 35 & $\circ$ & Continues & - & - \\
\hline & Nozzle's outlet angle & $\beta_{4}$ & 0.5 & 10 & $\circ$ & Continues & - & - \\
\hline & Safety factor & $\eta_{\text {safety }}$ & \multicolumn{2}{|c|}{1.5} & - & Continues & $\% 10$ & Normal \\
\hline & Catalyst bed porosity & $\varepsilon_{C B}$ & \multicolumn{2}{|c|}{0.34} & - & Continues & $\% 5$ & Normal \\
\hline
\end{tabular}

$\frac{L_{C C}}{D_{C C}}+\sigma_{\frac{L_{C C}}{D_{C C}}} \leq 2$

$$
M_{N_{i n}}+\sigma_{M_{N_{i n}}} \leq 0.3 \text {. }
$$

\subsubsection{Optimization results}

The monopropellant thruster Multi-Objective Multidisciplinary Design Optimization (MOMDO) and
Multi-Objective Robust Multi-disciplinary Design Optimization (MORMDO) results are presented in this section. In this study, the best design was selected based on the minimum distance from the Utopia point. In the minimum distance or knee point method, the point with the minimum distance from the Utopia point [37] was considered the appropriate answer.

The multi-objective MDO was performed in 
Table 5. Objective functions at the best point of Multi-Objective Multidisciplinary Design Optimization (MOMDO) and Multi-Objective Multidisciplinary Robust Design Optimization (MOMRDO) of the monopropellant thruster.

\begin{tabular}{ccccccccccc}
\hline Methods & $\boldsymbol{w}_{\boldsymbol{1}}$ & $\boldsymbol{w}_{\boldsymbol{2}}$ & $\boldsymbol{w}_{\mathbf{3}}$ & $\boldsymbol{I}_{\boldsymbol{s p}}$ & $\boldsymbol{\sigma}_{\boldsymbol{I}_{\boldsymbol{s} p}}$ & $\boldsymbol{M}_{\text {Total }}$ & $\boldsymbol{\sigma}_{\boldsymbol{M}}$ & $\boldsymbol{\Delta} \boldsymbol{P}$ & $\boldsymbol{\sigma}_{\boldsymbol{\Delta} \boldsymbol{P}}$ & Calculation time (s) \\
\hline MDO & 1 & 0 & 0 & 237.80 & - & 206.91 & - & 2.47 & - & 2598 \\
RMDO & 0.6 & 0.4 & 0 & 228.86 & 1.48 & 234.922 & 6.68 & 3.19 & 0.38 & 97653 \\
\hline
\end{tabular}

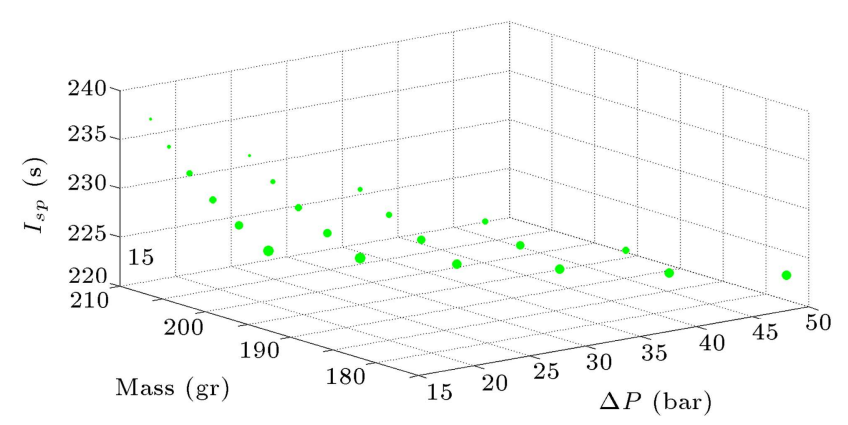

Figure 14. The Pareto front of the Multi-Objective Multidisciplinary Design Optimization (MOMDO) of the monopropellant thruster.

twenty-one iterations with an average calculation time of approximately 2708 seconds. The Pareto front of the MOMDO of the monopropellant thruster was presented in Figure 14. In this graph, each point represents one of the multi-objective optimization results and the diameter of these points represents the distance from the ideal point, so as the distance from the knee point increases; the diameter of these points also increases. According to the knee point criterion, the best design was obtained at the 21st point.

The results of the MORMDO of the monopropellant thruster are depicted as the Pareto front and the variation of the standard deviation of specific impulse, the standard deviation of the mass, the standard deviation of the total pressure drop of the system at different points of the design objective function and their histogram are shown in Figures 14 to 18. The average convergence time at any point of the Pareto front was about 11,1039 seconds. The best

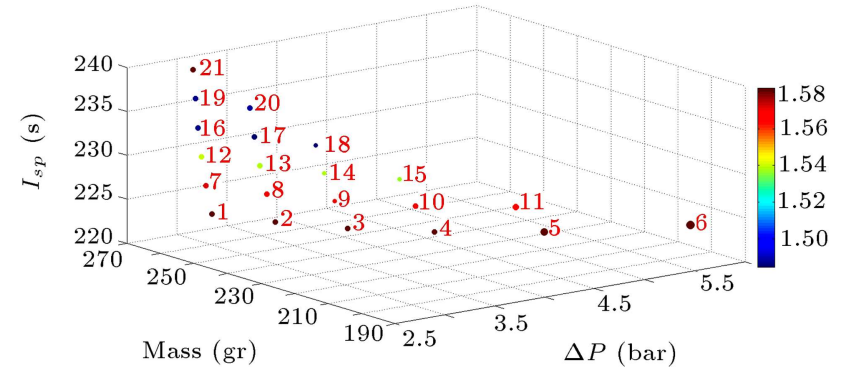

Figure 15. The Pareto front of Multi-Objective Multidisciplinary Robust Design Optimization (MOMRDO) of the monopropellant thruster and standard deviation of specific impulse. answer was at point 18 . By comparing the specific impulse of the non-deterministic optimal design with the specific impulse of the deterministic optimal design, an average decrease of $75.3 \%$ was obtained, while the mass decreased and pressure drop increased by $13.53 \%$ and $29.14 \%$, respectively.

The values of the objective functions, design variables, and optimization constraints at the best point of MOMDO and MORMDO of the monopropellant thruster are shown in Table 5 to Table 7 , respectively.

In both deterministic and non-deterministic opti-

Table 6. Design variables at the best point of Multi-Objective Multidisciplinary Design Optimization (MOMDO) and Multi-Objective Multidisciplinary Robust Design Optimization (MOMRDO) of the monopropellant thruster.

\begin{tabular}{cccccc}
\hline \multirow{2}{*}{ Variable } & \multicolumn{2}{c}{ Rang } & Unit & MDO & RMDO \\
\cline { 2 - 3 } & $\min$ & $\mathbf{m a x}$ & & & \\
\hline$P_{C C}$ & 5 & 20 & bar & 15.60 & 12.65 \\
$D_{C C}$ & 10 & 40 & $\mathrm{~mm}$ & 25 & 27.6 \\
$\alpha$ & 60 & 90 & $\circ$ & 87 & 80 \\
$\nu$ & 10 & 35 & $\circ$ & 30 & 30.5 \\
$n$ & 3 & 5 & $\#$ & 3 & 3 \\
$T_{0}$ & 250 & 350 & $\mathrm{~K}$ & 310 & 298.5 \\
$D_{P}$ & 0.5 & 2 & $\mathrm{~mm}$ & 1.25 & 0.91 \\
$\eta_{C C}$ & 0.85 & 0.97 & - & 0.87 & 0.92 \\
$\varepsilon_{N}$ & 2 & 100 & - & 65 & 58 \\
$\beta_{3}$ & 20 & 35 & $\circ$ & 29 & 25 \\
$\beta_{4}$ & 0.5 & 10 & $\circ$ & 4.5 & 4 \\
\hline
\end{tabular}

Table 7. Values of the constraints at the best point of Multi-disciplinary Design Optimization (MDO) and RMDO of the monopropellant thruster.

\begin{tabular}{cccc}
\hline Constraint & Unit & MDO & RMDO \\
\hline$P_{\text {in }}$ & bar & 17.47 & 15.9 \\
$G_{\text {Cat }}$ & $\mathrm{kg} / \mathrm{m}^{2}$ & 4.80 & 4.09 \\
$\frac{L_{C C}}{D_{C C}}$ & - & 1.65 & 1.43 \\
$M_{N_{\text {in }}}$ & - & 0.01 & 0.01 \\
\hline
\end{tabular}



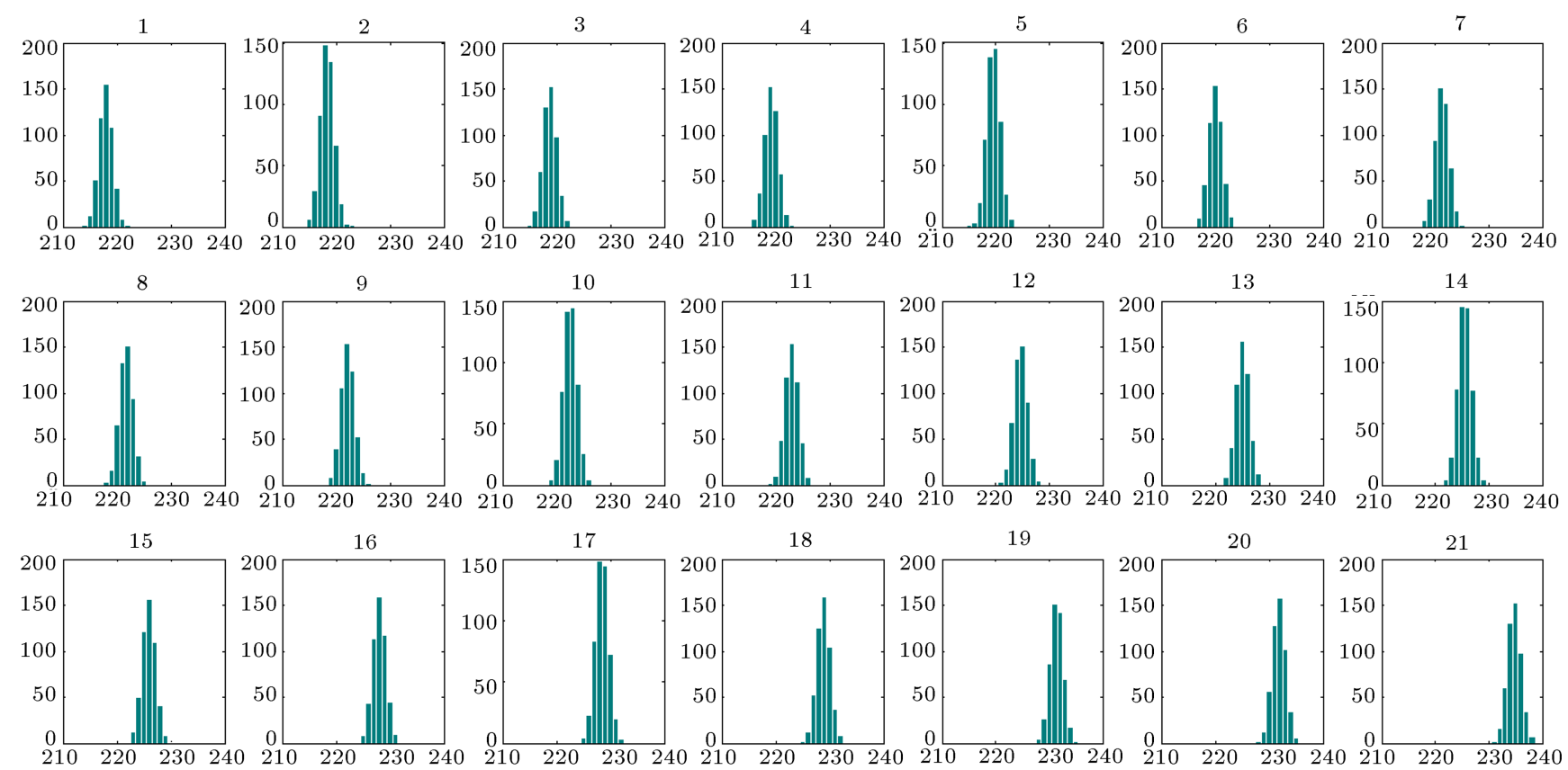

Figure 16. Multi-Objective Multidisciplinary Robust Design Optimization (MOMRDO) histogram of monopropellant thruster specific impulse at different points.

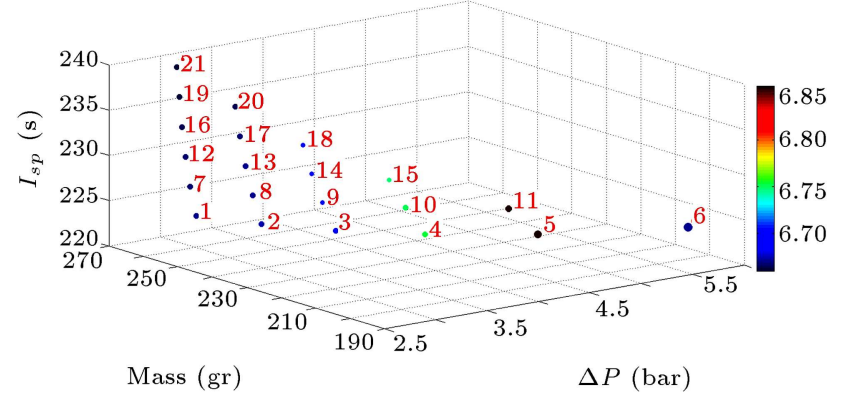

Figure 17. The Pareto front of Multi-Objective Multidisciplinary Robust Design Optimization (MOMRDO) of the monopropellant thruster and standard deviation of the mass.

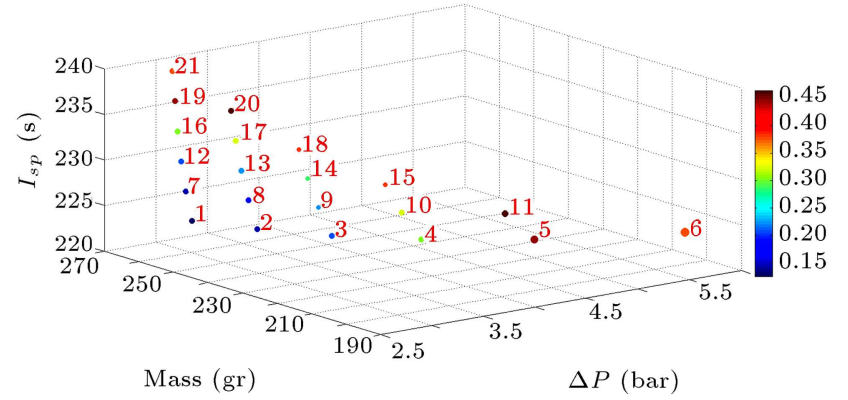

Figure 18. The Pareto front of Multi-Objective Multidisciplinary Robust Design Optimization (MOMRDO), standard deviation of the total pressure drop.

mization methods, all constraints were satisfied. Moreover, the results shown in Table 7 show that in addition to increasing the robustness of the design objectives, the application of the proposed algorithm also led to an increase in the robustness of the constraints.

\section{Conclusions}

In this study, a new Multi-Objective Robust Multidisciplinary Design Optimization (MORMDO) method based on a fast and efficient Uncertainty Analysis (UA) strategy was developed. The Bi-Level Integrated System Synthesis (BLISS) framework has been implemented as an Multidisciplinary Design Optimization (MDO). The calculation of the UA was done using Progressive Latin Hypercube Sampling (PLHS). In this strategy, the convergence of the UA determines the sample size.

The performance of the UA and Robust Design Optimization (RDO) algorithms was verified by characteristics consideration of optimization performance, sampling algorithm convergence, robust optimization and computational cost and also was validated by benchmark functions. The validation results showed that the application of the proposed method to Multidisciplinary Robust Design Optimization (MRDO) problems leads to a systematic reduction of the sample size which is required to obtain a good UA with suitable accuracy. The results of the evaluations performed in the sections of "Sampling convergence characteristics" and "Computational cost" indicated that the performance of progressive sampling was correct. On the other hand, in Monte Carlo Simulation (MCS) and LHS-based uncertainty analyses with fixed sample size, at least 100,000 and 1,000 samples were needed 
to ensure the convergence of the analysis. However, PLHS based UA converged with an average of 300 samples. This fact indicated a significant reduction in the computational cost of the PLHS based RDO.

In addition, the RDO of a hydrazine monopropellant thruster was defined as a case study of the proposed framework. Based on this, all subsystems (structure, injector plate, catalyst bed, and nozzle) were introduced and their governing equations were extracted. In the monopropellant optimization process, the maximum specific impulse $\left(I_{s p}\right)$ and minimum weight and pressure drop were defined as the objectives function of the RDO. Besides, the maximum feed pressure, maximum catalyst bed loading factor, catalyst bed aspect ratio, and nozzle input Mach number were proposed as design constraints. Two optimization problems including the deterministic and non-deterministic optimization, have been solved for this thruster. For multi-objective optimization, the Pareto front was extracted as optimal response, and the best optimum point was selected based on the minimum distance strategy. The results of the deterministic and non-deterministic optimization problems were compared to each other. By comparing the specific impulse of the non-deterministic optimal design with the specific impulse of the deterministic optimal design the average drop of $75.3 \%$ was obtained, while the mass and pressure drop has increased by $13.53 \%$ and $29.14 \%$, respectively.

The results of this study showed that the proposed method was a fast and efficient method for optimizing the design of the complex systems and that this approach can be used for other engineering applications as well.

\section{Supplementary data}

Supplementary data are available at:

http://scientiairanica.sharif.edu/jufile?ar_sfile $=146238$

\section{Nomenclature}

$\begin{array}{ll}D_{P} & \text { Pellet diameter } \\ D_{C C} & \text { Reaction chamber diameter } \\ H^{m} & \text { Unit } m \text { dimensional hypercube } \\ I_{s p} & \text { Thruster specific impulse } \\ K & \text { pre-exponential factor } \\ L_{C C} & \text { Reaction chamber Length } \\ M & \text { Mass } \\ m & \text { Number of design variables } \\ n & \text { Sample size, injector groves number } \\ P_{C C} & \text { Reaction chamber pressure } \\ S(n, m) & \text { Sample matrix }\end{array}$

$\begin{array}{ll}T_{0} & \text { Propellant inlet temperature } \\ t & \text { Thickness of the structure } \\ x & \text { Design variables } \\ y_{i, j} & \text { Auxiliary binary variables } \\ \alpha_{i n j} & \text { Propellant injection angle } \\ \beta_{i} & \text { Nozzle divergent angle } \\ \Delta P & \text { Thruster pressure drop } \\ \phi & \text { Robust optimization objective } \\ v & \text { Injector groves angle } \\ \tau & \text { Population correction factor }\end{array}$

\section{References}

1. Li, W., Xiao, M., and Gao, L. "Improved collaboration pursuing method for multidisciplinary robust design optimization", Structural and Multidisciplinary Optimization, 59(6), pp. 1949-1968 (2019).

2. Sobieszczanski-Sobieski, J. and Haftka, R.T. "Multidisciplinary aerospace design optimization: survey of recent developments", Structural Optimization, 14(1), pp. 1-23 (1997).

3. Hwang, J.T., Jain, A.V., and Ha, T.H. "Large-scale multidisciplinary design optimization-review and recommendations", in AIAA Aviation 2019 Forum, p. 3106 (2019).

4. Wang, X., Liu, Y., Sun, W., Song, X., and Zhang, J. "Multidisciplinary and multifidelity design optimization of electric vehicle battery thermal management system", Journal of Mechanical Design, 140(9), p. 094501 (2018).

5. Schappler, M., Tappe, S., and Ortmaier, T. "Exploiting dynamics parameter linearity for design optimization in combined structural and dimensional robot synthesis", in IFToMM World Congress on Mechanism and Machine Science, Springer, pp. 1949-1958 (2019).

6. Fowlkes, W.Y., Creveling, C.M., and Derimiggio, J. "Engineering methods for robust product design: using Taguchi methods in technology and product development", Addison-Wesley Reading, MA (1995).

7. Wu, F., Ma, Y., and Wang, J. "Robust parameter design based on response surface model under considering measurement errors", Scientia Iranica, Trans. E, 28(4) (2021). DOI: $10.24200 /$ sci.2019.50622.1793

8. McAllister, C.D. and Simpson, T.W. "Multidisciplinary robust design optimization of an internal combustion engine", Journal of Mechanical Design, 125(1), pp. 124-130 (2003).

9. Yao, W., Guo, J., Chen, X., and Van Tooren, M. "Utilizing uncertainty multidisciplinary design optimization for conceptual design of space systems", in 8th Annual Conference on Systems Engineering Research, CSER 2010, Hoboken, NJ, USA, 17-19 (March 2010).

10. Xu, H., Li, W., Li, M., Hu, C., Zhang, S., and Wang, $\mathrm{X}$. "Multidisciplinary robust design optimization based 
on time-varying sensitivity analysis", Journal of $\mathrm{Me}$ chanical Science and Technology, 32(3), pp. 1195-1207 (2018).

11. Schuëller, G.I. and Jensen, H.A. "Computational methods in optimization considering uncertainties-an overview", Computer Methods in Applied Mechanics and Engineering, 198(1), pp. 2-13 (2008).

12. Iooss, B. and Lemaitre, P. "A review on global sensitivity analysis methods", in Uncertainty Management in Simulation-Optimization of Complex Systems, Springer, pp. 101-122 (2015).

13. Robinson, D. and Atcitty, C. "Comparison of quasiand pseudo-Monte Carlo sampling for reliability and uncertainty analysis", in 40th Structures, Structural Dynamics, and Materials Conference and Exhibit, St. Louis, MO, U.S.A, p. 1589 (1999).

14. Tong, C. "Refinement strategies for stratified sampling methods", Reliability Engineering \& System Safety, 91(10-11), pp. 1257-1265 (2006).

15. ElRafey, A. and Wojtusiak, J. "Recent advances in scaling-down sampling methods in machine learning", Wiley Interdisciplinary Reviews: Computational Statistics, 9(6), p. e1414 (2017).

16. Liu, Z., Li, W., and Yang, M. "Two general extension algorithms of Latin hypercube sampling", Mathematical Problems in Engineering, 2015 (2015).

17. Williamson, D. "Exploratory ensemble designs for environmental models using k-extended Latin hypercubes", Environmetrics, 26(4), pp. 268-283 (2015).

18. Qian, P.Z. "Sliced Latin hypercube designs", Journal of the American Statistical Association, 107(497), pp. 393-399 (2012).

19. Ba, S., Myers, W.R., and Brenneman, W.A. "Optimal sliced Latin hypercube designs", Technometrics, 57(4), pp. $479-487$ (2015).

20. Sobieszczanski-Sobieski, J., Agte, J., and Sandusky, R. "Bi-level integrated system synthesis (bliss)", Langley Research Center, Hampton, Virginia, NASA Technical Report TM-1998-208715, 9 (1998).

21. Vuyst De, F., Multidisciplinary Design Optimization in Computational Mechanics, chapter PDE Metamodeling using Principal Component Analysis, Ed: Wiley ISTE, April (2010).

22. Hu, X., Chen, X., Parks, G.T., and Yao, W. "Review of improved Monte Carlo methods in uncertainty-based design optimization for aerospace vehicles", Progress in Aerospace Sciences, 86, pp. 20-27 (2016).

23. Kroese, D.P., Taimre, T., and Botev, Z.I., Handbook of Monte Carlo Methods, John Wiley \& Sons (2013).

24. Van Nguyen, N., Lee, J.-W., Lee, Y.-D., and Park, H.-U. "A multidisciplinary robust optimisation framework for UAV conceptual design", The Aeronautical Journal, 118(1200), pp. 123-142 (2014).
25. Othman, M.F., Silva, G.H., Cabral, P.H., Prado, A.P. Pirrera, A., and Cooper, J.E. "A robust and reliabilitybased aeroelastic tailoring framework for composite aircraft wings", Composite Structures, 208, pp. 101113 (2019).

26. Yi, S.-I., Shin, J.-K., and Park, G. "Comparison of MDO methods with mathematical examples", Structural and Multidisciplinary Optimization, 35(5), pp. 391-402 (2008).

27. Perez, R., Liu, H., and Behdinan, K. "Evaluation of multidisciplinary optimization approaches for aircraft conceptual design", in 10th AIAA/ISSMO Multidisciplinary Analysis and Optimization Conference, p. 4537 (2004).

28. Bayvel, L. and Orzechowski, Z., Liquid Atomization, Combustion: An International Series, Taylor \& Francis (1993).

29. Hwang, C.H., Lee, S.N., Baek, S.W., Han, C.Y., Kim, S.K., and Yu, M.J. "Effects of catalyst bed failure on thermochemical phenomena for a hydrazine monopropellant thruster using $\mathrm{Ir} / \mathrm{Al}_{2} \mathrm{O}_{3}$ catalysts", Industrial \& Engineering Chemistry Research, 51(15), pp. 5382-5393 (2012).

30. Naseh, H., Meibody, M.N.P., Hosseini Anari, H., and Ommi, F. "Numerical-parametrical analysis on the hydrogen peroxide catalyst bed for space monopropellant thruster applications", (in Persian), Journal of Applied Research of Chemical-Polymer Engineering, 1(2), pp. 17-30 (2018).

31. Meibody, M.N.P., Naseh, H., and Ommi, F. "A kriging based multi objective gray wolf optimization for hydrazine catalyst bed", Engineering Solid Mechanics, 7(3), pp. 179-192 (2019).

32. Naseh, H., Meibody, M.N.P., and Ommi, F. "Sensitivity analysis based on progressive LHS applied to hydrazine catalyst bed design", JSST, 12(4), pp. 3546 (2019).

33. Meibody, M.N.P., Naseh, H., and Ommi, F. "Progressive Latin Hypercube sampling-based robust design optimisation (PLHS-RDO)", Australian Journal of Mechanical Engineering, pp. 2204-2253 (2020). DOI: 10.1080/14484846.2020.1733177

34. Ghassemi, H., Meibody, M.N.P., and Asghari, I. "Experimental investigation on specific velocity of hydrogenperoxid monopropellant thruster", Presented at the 11th Iranian Aerospace Society Conference, Tehran, Iran, March 1-3 (2011).

35. Pasini, A., Torre, L., Romeo, L., Cervone, A., and d'Agostino, L. "Performance characterization of pellet catalytic beds for hydrogen peroxide monopropellant rockets", Journal of Propulsion and Power, 27(2), pp. 428-436 (2011).

36. Huzel, D.K., Modern Engineering for Design of LiquidPropellant Rocket Engines, AiA A (1992). 
37. Babaei, A.R., Setayandeh, M.R., and Farrokhfal, H. "Aircraft robust multidisciplinary design optimization methodology based on fuzzy preference function", Chinese Journal of Aeronautics, 31(12), pp. 2248-2259 (2018).

\section{Biographies}

Mohammad Nadafi Pour Meibody received his MSc in Aerospace Engineering from Iran University of Science and Technology, Iran in 2011. His MSc research involved design, manufacturing and testing of the hybrid motor. He received his $\mathrm{PhD}$ in Aerospace Engineering from Aerospace Research Institute (ARI), Ministry of Science, Research and Technology (MSRT), Tehran, Iran in 2020. His research interests include the system engineering, Robust Design Optimization (RDO) and Uncertainty Analysis (UA) in Multidisciplinary Design Optimization (MDO).

Hassan Naseh received his MSc in Aerospace Engineering from the K. N. Toosi University of Tech- nology, Iran in 2007. His MSc research involved the conceptual design of aerospace vehicles. His research interests include the space system engineering and Multi-disciplinary Design Optimization (MDO). He received a $\mathrm{PhD}$ in Aerospace engineering from $\mathrm{K}$. N. Toosi University of Technology, Iran 2014. He is a faculty member (Assistant Professor) in Aerospace Research Institute (ARI), Ministry of Science, Research and Technology (MSRT), Tehran, Iran, since 2014.

Fathollah Ommi received his MSc in Mechanical Engineering from Tarbiat Modares University, Iran, 1989. His MSc research involved energy transfer. He received his $\mathrm{PhD}$ in Mechanical Engineering in the field of Aerospace engineering from University of Technology, Moscow, Russia in 1996. He is a faculty member (Full Professor) in the department of mechanical engineering, Tarbiat Modares University, Tehran, Iran. His research interests include the propulsion, combustion, spray and atomization, experimental study and gas turbine theory. 